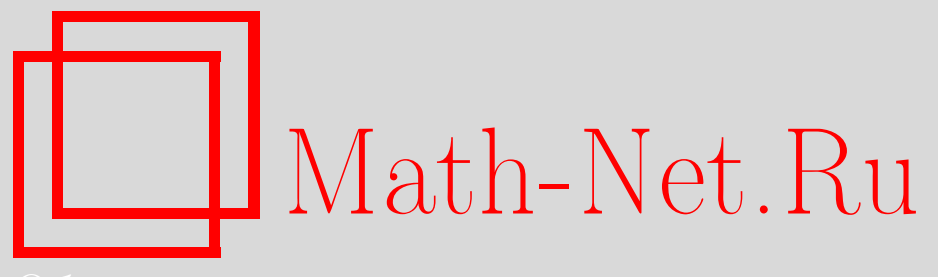

G. P. Chistyakov, F. Götze, Moderate deviations for Student's statistic, Теория вероятн. и ее примен., 2002, том 47, выпуск 3, 518-532

DOI: https://doi.org/10.4213/tvp3690

Использование Общероссийского математического портала MathNet.Ru подразумевает, что вы прочитали и согласны с пользовательским соглашением

http://www . mathnet.ru/rus/agreement

Параметры загрузки:

IP : 34.229 .108 .108

26 апреля 2023 г., 17:28:25

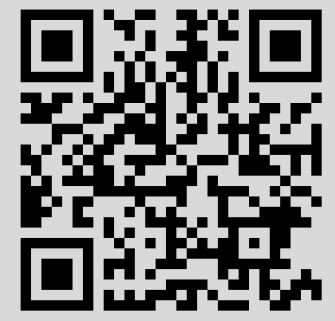




\section{MODERATE DEVIATIONS FOR STUDENT'S STATISTIC ${ }^{1)}$}

Для самонормированных сумм, скажем, $S_{n} / V_{n}$, при условиях симметрии рассматриваются зоны Линника, где отношение $\mathbf{P}\left\{S_{n} / V_{n} \geqslant x\right\} /(1-\Phi(x))$ сходится к 1 , и устанавливаются оптимальные оценки для остаточных членов при этой сходимости.

Ключевые слова и фразы: зоны Линника, самонормированная сумма, $t$-статистика, умеренные уклонения, неравномерные оценки.

1. Introduction and results. Let $X, X_{1}, X_{2}, \ldots$ denote independent identically distributed (i.i.d.) random variables such that $\mathbf{E} X=0$ and $0<\sigma^{2}=\mathbf{E} X^{2}<\infty$. Write

$$
S_{n}=\sum_{j=1}^{n} X_{j} \quad \text { and } \quad V_{n}^{2}=\sum_{j=1}^{n} X_{j}^{2}
$$

Define the self-normalized sum as $S_{n} / V_{n}$, if $V_{n} \neq 0$, and 0 if $V_{n}=0$.

Let $\Lambda(n)$ denote a nondecreasing function satisfying $\Lambda(n) \rightarrow \infty$ as $n \rightarrow \infty$. The interval $[0, \Lambda(n)]$ is called zone of normal convergence if

$$
\frac{1-\mathbf{P}\left\{S_{n}<x \sigma \sqrt{n}\right\}}{1-\Phi(x)} \longrightarrow 1, \quad \frac{\mathbf{P}\left\{S_{n}<-x \sigma \sqrt{n}\right\}}{\Phi(-x)} \longrightarrow 1
$$

uniformly in $x \in[0, \Lambda(n)]$, where $\Phi$ denotes the standard Gaussian distribution function. It is well known, assuming that the condition $\beta=\mathbf{E}|X|^{3}<\infty$ holds, that the Berry-Esseen bound implies (1.1) with $\Lambda(n)=(1-\varepsilon) \sqrt{\ln n}$, for $0<\varepsilon<1$. In this case the zone of normal convergence is of logarithmic size and is rather narrow. Consider the condition

$$
\mathbf{E} \exp \left\{\left|X_{1}\right|^{4 \alpha /(2 \alpha+1)}\right\}<\infty
$$

with some $\alpha$ such that $0<\alpha \leqslant \frac{1}{6}$. Linnik [13] (see as well [12]) proved the following result. For any function $\rho(n)$ with $\lim _{n \rightarrow \infty} \rho(n)=\infty$ the relation (1.1) holds with $\Lambda(n)=n^{\alpha} / \rho(n)$. Furthermore, if (1.1) holds with

*Физико-технический институт низких температур им. Б. И. Веркина НАН Украины, пр. Ленина, 47, Харьков, 61164 Харьков; e-mail: chistyakov@ilt.kharkov.ua

**Fakultät für Mathematik, Universität Bielefeld, Postfach 100131, 33501 Bielefeld 1, Germany; e-mail: goetze@mathematik.uni-bielefeld.de

1) Research of both authors supported by the SFB 343 in Bielefeld; research of the second author supported also by INTAS 99-00317, RFBR-DFG 99-01-04027. 
$\Lambda(n)=n^{\alpha} \rho(n)$, then the condition (1.2) is fulfilled. This result of Linnik shows that for deterministically normalized sums the existence of exponential moments is a natural condition for the existence of power type zones of normal convergence.

Giné, Götze, and Mason [8] noticed that the tails of $S_{n} / V_{n}$ are uniformly sub-Gaussian in cases when the sequence $S_{n} / V_{n}$ is stochastically bounded. This observation suggests that Linnik type results for self-normalized sums have to hold under conditions considerably weaker than (1.2). Shao [19] established that for self-normalized sums Linnik type results hold under the more natural condition of the existence of the third moment $\beta<\infty$. In this paper under symmetry conditions we shall obtain optimal explicit bounds for the remainders in relations (1.1) for the self-normalized sums (see Theorem 1 and Corollary 1 below). The bounds will depend on $n, x, \sigma, \beta$ only. In Theorem 2 we extend the results to the non-i.i.d. situation. In this more general setting the bounds depend on $x$ and on Lyapunov's ratio $L_{n}$ only. Below we shall denote by $\theta$ different real valued quantities such that $|\theta| \leqslant 1$. The notation $c, c_{1}, c_{2}, \ldots$ will be used throughout for absolute positive constants. By $c$ we denote different constants in different (or even in the same) formulae. The symbols $c_{1}, c_{2}, \ldots$ are applied for concrete constants. We shall prove the following relations:

$$
\begin{aligned}
& \frac{\mathbf{P}\left\{S_{n} / V_{n}>x\right\}}{1-\Phi(x)}=1+c \theta r_{n}(x), \quad \text { for } \quad x \geqslant 0, \\
& \frac{\mathbf{P}\left\{S_{n} / V_{n}<x\right\}}{\Phi(x)}=1+c \theta r_{n}(x), \quad \text { for } \quad x \leqslant 0,
\end{aligned}
$$

where $r_{n}(x)$ is a function which we have to specify. Notice that for $|x|>\sqrt{n}$ the left-hand sides of (1.3) and (1.4) are equal to zero since $\mathbf{P}\left\{\left|S_{n}\right| / V_{n}>\right.$ $|x|\}=0$.

Theorem 1. Assume that $X$ is a symmetric random variable such that $\sigma^{2}=1$ and $\beta<\infty$. Then relations (1.3) and (1.4) hold with

$$
r_{n}(x)=\left\{\begin{array}{llr}
\frac{\left(|x|^{3}+1\right) \beta}{\sqrt{n}}, & \text { for } & 0 \leqslant|x| \leqslant \frac{n^{1 / 6}}{\beta^{1 / 3}} \\
1, & \text { for } & |x|>\frac{n^{1 / 6}}{\beta^{1 / 3}} .
\end{array}\right.
$$

Write

$$
\begin{array}{ll}
G(x, l)=\sup \mathbf{P}\left\{\frac{S_{n}}{V_{n}}>x\right\}, & x \geqslant 0, \\
G(x, l)=\sup \mathbf{P}\left\{\frac{S_{n}}{V_{n}}<x\right\}, & x \leqslant 0,
\end{array}
$$

where the supremum is taken over all symmetric $X$ such that the Lyapunov ratio $L_{n}:=\beta /\left(\sigma^{3} \sqrt{n}\right)$ is fixed, that is, $L_{n}=l$. Let $\rho(l)$ be a function such 
that $\rho(l) \geqslant 1$. Consider the relations

$$
\begin{aligned}
\frac{G(x, l)}{1-\Phi(x)} & =1+c \theta\left(l+\frac{1}{\rho(l)}\right), \quad \text { for } \quad x \geqslant 0, \\
\frac{G(x, l)}{\Phi(x)} & =1+c \theta\left(l+\frac{1}{\rho(l)}\right), \quad \text { for } \quad x \leqslant 0 .
\end{aligned}
$$

Corollary 1. Under the assumptions of Theorem 1 relations (1.6) and (1.7) hold for $|x| \leqslant 1 /(l \rho(l))^{1 / 3}$.

In particular, Corollary 1 yields

$$
\frac{\mathbf{P}\left\{S_{n} / V_{n}>x\right\}}{1-\Phi(x)}=1+o(1)
$$

uniformly for $x=o\left(n^{1 / 6}\right)$. To see this it suffices to choose an appropriate function $\rho$.

For $|x| \leqslant L_{n}^{-1 / 3}$ estimates (1.3) and (1.4) with $r_{n}(x)$ from Theorem 1 are exact in the following sense.

Proposition. Let $\left\{b_{n}\right\}_{n=1}^{\infty}$ be a sequence of real numbers such that $b_{n} \geqslant 1$ and $b_{n} / \sqrt{n} \rightarrow 0$ as $n \rightarrow \infty$. Then for every fixed $x \in\left(0, n^{1 / 6} / b_{n}^{1 / 3}\right]$ there exist symmetric random variables $X=X(x, n)$ and absolute positive constants $c, c_{1}, c_{2}$ such that $\mathbf{E} X^{2}=1, c_{1} b_{n} \leqslant \mathbf{E}|X|^{3} \leqslant c_{2} b_{n}$, and

$$
\frac{\mathbf{P}\left\{S_{n} / V_{n}>x\right\}}{1-\Phi(x)} \leqslant 1-c\left(x^{3}-c\left(x^{2}+1\right)\right) \frac{b_{n}}{\sqrt{n}} .
$$

This inequality holds for sufficiently large $\sqrt{n} / b_{n} \geqslant c$.

Let us discuss some results for self-normalized sums related to the central limit theorem (CLT) and their refinements. Self-normalized sums satisfy the CLT provided that $X$ belongs to the domain of attraction of a normal law [15], [4]). Griffin and Mason [9] proved that the condition is necessary in the symmetric case. Giné, Götze, and Mason [8] proved that the condition is necessary for nonsymmetric $X$ as well. Egorov [6] established necessary and sufficient conditions in the non-i.i.d. symmetric case. These results indicate that the self-normalization may improve the limiting behaviour of sums.

Berry-Esseen bounds of increasing generality and accuracy were obtained in [10], [22], [21], [1], [2]. The paper [1] contains bounds which imply, in particular, sufficient conditions for the CLT. The bounds extend to the non-i.i.d. case, see [2]. The bounds of the last two papers imply our results for $|x| \leqslant c$.

Sub-Gaussian type behaviour of tails related to self-normalized sums was observed in [14]. Giné, Götze, and Mason [8] showed that the tails of $S_{n} / V_{n}$ are uniformly sub-Gaussian in the case when a sequence $S_{n} / V_{n}$, $n \geqslant 1$, is stochastically bounded. Shao ([18]-[20]) considered large and moderate deviations. In particular, he proved (1.8) in the zone $x=o\left(n^{1 / 6}\right)$. 
Wang and Jing [23] proved a bound for symmetric independent nonidentically distributed random variables. Under the conditions of Theorem 1 it implies (1.3) and (1.4) in the i.i.d. case with

$$
r_{n}(x)= \begin{cases}L_{n}, & \text { for }|x| \leqslant 1 \\ |x|^{4} L_{n}, & \text { for } 1 \leqslant|x| \leqslant 5^{-1} L_{n}^{-1 / 3} \\ |x|, & \text { for }|x|>5^{-1} L_{n}^{-1 / 3}\end{cases}
$$

Notice that the function $r_{n}(x)$ in (1.9) contains for $|x| \geqslant 1$ an extra factor $|x|$, compared to $r_{n}(x)$ from Theorem 1. Wang and Jing [23] proved as well a bound of $r_{n}(x)$ in (1.3) and (1.4) for nonsymmetric i.i.d. random variables. Chistyakov and Götze [3] improved their results and considered even more general situation of non-i.i.d. random variables (see Theorems 2, 3, and 4 in [3]). In particular, the Wang and Jing bound for nonsymmetric i.i.d. random variables differs from the bound of Theorem 2 (ibid.) by an extra factor $\exp \left\{\varepsilon x^{2}\right\}, \varepsilon>0$.

Now let us consider the case of non-i.i.d. random variables. Henceforth $X_{1}, \ldots, X_{n}$ denote random variables such that

$$
\mathbf{E} X_{j}=0, \quad 0<\sigma_{j}^{2}=\mathbf{E} X_{j}^{2}<\infty, \quad \text { and } \quad \beta_{j}=\mathbf{E}\left|X_{j}\right|^{3}
$$

for all $j$. Write

$$
B_{n}^{2}=\sum_{j=1}^{n} \sigma_{j}^{2}, \quad A_{n}=\sum_{j=1}^{n} \beta_{j}, \quad L_{n}=\frac{A_{n}}{B_{n}^{3}} .
$$

Theorem 2. Let $X_{1}, \ldots, X_{n}$ be symmetric independent random variables such that $L_{n}<\infty$. Then relations (1.3) and (1.4) hold with

$$
r_{n}(x)=\left\{\begin{array}{lrr}
\left(|x|^{3}+1\right) L_{n}, & \text { for } & 0 \leqslant|x| \leqslant L_{n}^{-1 / 3} \\
1, & \text { for } & |x|>L_{n}^{-1 / 3} .
\end{array}\right.
$$

Corollary 2. Let $G(x, l)$ be defined by (1.5) with supremum taken over all symmetric independent nonidentically distributed random variables $X_{1}, \ldots, X_{n}$ such that $L_{n}=l$. Then under the assumptions of Theorem 2 relations (1.6) and (1.7) are valid for $|x| \leqslant 1 /(l \rho(l))^{1 / 3}$.

Note that Theorem 1 is an obvious consequence of Theorem 2. We omit the evident proofs of Corollaries 1 and 2. Consider Student's $t$-statistic $T_{n}$ defined by

$$
T_{n}=\frac{1}{\sqrt{n}} \sum_{j=1}^{n} X_{j} /\left(\frac{1}{n-1} \sum_{j=1}^{n}\left(X_{j}-\bar{X}_{n}\right)^{2}\right)^{1 / 2},
$$

where $\bar{X}_{n}=S_{n} / n$. It is well known (see [5]) that, for $x \geqslant 0$,

$$
\mathbf{P}\left\{T_{n}>x\right\}=\mathbf{P}\left\{\frac{S_{n}}{V_{n}}>\left(\frac{n x^{2}}{n+x^{2}-1}\right)^{1 / 2}\right\} .
$$


Denote $\Delta_{T_{n}}(x)=\left|\mathbf{P}\left\{T_{n}<x\right\}-\Phi(x)\right|$. With the help of (1.10) we obtain the following nonuniform bounds and Cramér large (moderate) deviations for $T_{n}$ as an immediate consequence of Theorem 2 .

Theorem 3. Under the assumptions of Theorem 2, we have, for all positive integers $n$ and $x \geqslant 0$,

$$
\Delta_{T_{n}}(x) \leqslant c \min \left\{\left(x^{3}+1\right) L_{n}, 1\right\}\left(1-\Phi\left(\left(\frac{n x^{2}}{n+x^{2}-1}\right)^{1 / 2}\right)\right) .
$$

Theorem 2 and Theorem 3 are consequences of Theorem 4 which shows that the preceding results hold for wide classes of nonindependent random variables $X_{1}, \ldots, X_{n}$.

Theorem 4. Let $X_{1}, \ldots, X_{n}$ be random variables such that the distributions of the random vectors $\left(\delta_{1} X_{1}, \ldots, \delta_{n} X_{n}\right)$ coincide for all values of $\delta_{j}$, where $\delta_{j}= \pm 1, j=1, \ldots, n$, and $L_{n}<\infty$. Then, for all $x \geqslant 0$,

$$
\begin{aligned}
&\left|\mathbf{P}\left\{\frac{S_{n}}{V_{n}}<x\right\}-\Phi(x)\right| \\
& \leqslant c \min \left\{1,\left(x^{3}+1\right)\left(L_{n}+\mathbf{P}\left\{V_{n}^{2}<\frac{B_{n}^{2}}{2}\right\}\right)\right\}(1-\Phi(x)), \\
& \Delta_{T_{n}}(x) \leqslant c \min \left\{1,\left(x^{3}+1\right)\left(L_{n}+\mathbf{P}\left\{V_{n}^{2}<\frac{B_{n}^{2}}{2}\right\}\right)\right\} \\
& \times\left(1-\Phi\left(\left(\frac{n x^{2}}{n+x^{2}-1}\right)^{1 / 2}\right)\right) .
\end{aligned}
$$

To prove Theorem 4 we apply the saddle-point method to the inversion formula for $\mathbf{P}\left\{S_{n} / V_{n}<x\right\}$ and with the help of this method obtain the optimal result in the case of symmetric random variables. Our method of the proof of Theorem 4 essentially differs from the methods used by Shao [18], [20], and Wang and Jing [23].

2. Proof of Theorem 2, Theorem 4, and Proposition. In the first step we shall prove Theorem 4 assuming, without loss of generality, that $x>0$.

We have, by the inversion formula (see [16, Ch. I, §3],

$$
\begin{aligned}
\mathbf{P} & \left\{\frac{S_{n}}{V_{n}}<N\right\}-\mathbf{P}\left\{\frac{S_{n}}{V_{n}}<x\right\} \\
& =\lim _{T \rightarrow \infty} \frac{1}{2 \pi} \int_{-T}^{T} \frac{e^{-i x t}-e^{-i N t}}{i t} \mathbf{E} e^{i t S_{n} / V_{n}} d t
\end{aligned}
$$

for all real $x, N$. Denote by $P_{1, \ldots, n}$ the distribution of the random vector $\left(X_{1}, \ldots, X_{n}\right)$ and rewrite the preceding formula, using the well- 
known relation

$$
\mathbf{E} e^{i t S_{n} / V_{n}}=\int_{\mathbf{R}^{n}} \prod_{j=1}^{n} \cos \frac{t x_{j}}{v_{n}} d P_{1, \ldots, n}, \quad t \in \mathbf{C},
$$

where $v_{n}=\left\{x_{1}^{2}+\cdots+x_{n}^{2}\right\}^{1 / 2}$ and $\mathbf{C}$ is the open complex plane. Here and in the sequel we shall assume that $\frac{0}{0}=0$. Let $D$ denote Borel's set of $\mathbf{R}^{n}$. Denote

$$
I(x, N, D)=\int_{D} \lim _{T \rightarrow \infty} \frac{1}{2 \pi} \int_{-T}^{T} \frac{e^{-i x t}-e^{-i N t}}{i t} \prod_{j=1}^{n} \cos \frac{t x_{j}}{v_{n}} d t d P_{1, \ldots, n} .
$$

Then with the help of (2.2) and Lebesgue's dominated convergence theorem we obtain from $(2.1)$

$$
1-\mathbf{P}\left\{\frac{S_{n}}{V_{n}}<x\right\}=\lim _{N \rightarrow \infty} I\left(x, N, \mathbf{R}^{n} \backslash D\right)+\lim _{N \rightarrow \infty} I(x, N, D) .
$$

The function under the integral sign on the right-hand side of (2.1) is a regular function in $t$ for all values of $t \in \mathbf{C}$ and its absolute value is bounded in each strip $|\operatorname{Im} t|<a(a>0)$. Using Cauchy's theorem we obtain the formula

$$
\begin{aligned}
\lim _{T \rightarrow \infty} & \frac{1}{2 \pi} \int_{-T}^{T} \frac{e^{-i x t}-e^{-i N t}}{i t} \prod_{j=1}^{n} \cos \frac{t x_{j}}{v_{n}} d t \\
= & e^{-x^{2}} \lim _{T \rightarrow \infty} \frac{1}{2 \pi} \int_{-T}^{T} \frac{e^{-i x t}}{x+i t} \prod_{j=1}^{n} \cos \frac{(t-i x) x_{j}}{v_{n}} d t \\
& -y e^{-N x} \lim _{T \rightarrow \infty} \frac{1}{2 \pi} \int_{-T}^{T} \frac{e^{-i N t}}{x+i t} \prod_{j=1}^{n} \cos \frac{(t-i x) x_{j}}{v_{n}} d t .
\end{aligned}
$$

It is easy to see that

$$
\sup _{\left(x_{1}, \ldots, x_{n}\right) \in \mathbf{R}^{n}} \sup _{N \in \mathbf{R}}\left|\lim _{T \rightarrow \infty} \int_{-T}^{T} e^{-i N t} \frac{1}{x+i t} \prod_{j=1}^{n} \cos \frac{(t-i x) x_{j}}{v_{n}} d t\right|<\infty .
$$

Indeed, the product of the second and third factor under the integral sign is the Fourier transform of a function $f$ such that $0 \leqslant f\left(u ; x_{1}, \ldots, x_{n}\right) \leqslant A<\infty$, where $A$ does not depend on $x_{1}, \ldots, x_{n}$ and $N$.

From the last two relations we deduce the following key formula

$$
\lim _{N \rightarrow \infty} I(x, N, D)=e^{-x^{2}} \int_{D} \prod_{j=1}^{n} \operatorname{ch} \frac{x x_{j}}{v_{n}} \cdot \lim _{T \rightarrow \infty} I(x, T) d P_{1, \ldots, n}
$$

for all $x>0$, where

$$
I(x, T)=\frac{1}{2 \pi} \int_{-T}^{T} \frac{e^{-i x t}}{x+i t} \prod_{j=1}^{n} \psi_{j}(t ; x) d t
$$

and $\psi_{j}(t ; x)=\cos \left\{(t-i x) x_{j} / v_{n}\right\} / \operatorname{ch}\left\{x x_{j} / v_{n}\right\}$. 
In the sequel we use (2.3) and (2.4) with $D=Q$, where $Q$ is the set of points $\left(x_{1}, \ldots, x_{n}\right) \in \mathbf{R}^{n} \backslash\{\mathbf{0}\}$ such that

$$
L=L\left(x_{1}, \ldots, x_{n}\right)=\frac{\left|x_{1}\right|^{3}+\cdots+\left|x_{n}\right|^{3}}{v_{n}^{3}} \leqslant \frac{1}{8(x+1)^{3}} .
$$

To estimate $I(x, N, Q)$ we need the following lemmas.

Lemma 1. Let $X_{1}, \ldots, X_{n}$ be independent random variables such that $\mathbf{E} X_{j}=0, \sigma_{j}^{2}>0, \beta_{j}<\infty, j=1, \ldots, n$. Then, for any $\varepsilon>0$,

$$
\mathbf{P}\left\{\left|V_{n}^{2}-B_{n}^{2}\right|>\varepsilon B_{n}^{2}\right\} \leqslant \frac{4}{\varepsilon^{3 / 2}} L_{n} .
$$

$\mathrm{P}$ r o o f. To prove (2.6) we use the von Bahr and Esseen result (see [16, Ch. III, $\S 5])$. Let $Y_{1}, \ldots, Y_{n}$ be independent random variables with zero means and finite absolute moments of order $p(1 \leqslant p \leqslant 2)$. Then

$$
\mathbf{E}\left|\sum_{k=1}^{n} Y_{k}\right|^{p} \leqslant\left(2-\frac{1}{n}\right) \sum_{k=1}^{n} \mathbf{E}\left|Y_{k}\right|^{p} .
$$

We obtain with the help of Chebyshev's inequality and the previous bound

$$
\begin{aligned}
\mathbf{P}\left\{\left|V_{n}^{2}-B_{n}^{2}\right|>\varepsilon B_{n}^{2}\right\} & \leqslant\left(\varepsilon B_{n}^{2}\right)^{-3 / 2} \mathbf{E}\left|\sum_{j=1}^{n}\left(X_{j}^{2}-\sigma_{j}^{2}\right)\right|^{3 / 2} \\
& \leqslant 2 \sum_{j=1}^{n}\left(\varepsilon B_{n}^{2}\right)^{-3 / 2} \mathbf{E}\left|X_{j}^{2}-\sigma_{j}^{2}\right|^{3 / 2} \leqslant 4 \varepsilon^{-3 / 2} L_{n}
\end{aligned}
$$

and (2.6) is proved.

Lemma 2. Let $X_{1}, \ldots, X_{n}$ denote independent random variables under the assumptions of Lemma 1 . Denote by $f_{n}(t)$ the characteristic function of the random variable $B_{n}^{-1} \sum_{j=1}^{n} X_{j}$. Then

$$
\left|f_{n}(t)-e^{-t^{2} / 2}\right| \leqslant 16 L_{n}|t|^{3} e^{-t^{2} / 3}, \quad|t| \leqslant \frac{1}{4 L_{n}} .
$$

The proof of this lemma see in [16, Ch. V, §2].

Lemma 3. Let $X$ be a random variable such that $\mathbf{E} e^{y X}<\infty$ for $0 \leqslant$ $y \leqslant a$. Then, for $x>0$ and $0 \leqslant y \leqslant a$,

$$
1-\mathbf{P}\{X<x\}=e^{-y x} \lim _{T \rightarrow \infty} \frac{1}{2 \pi} \int_{-T}^{T} \frac{e^{-i x t}}{y+i t} \mathbf{E} e^{(y+i t) X} d t .
$$

The proof of this lemma is similar to the proof of formula (2.4) and hence will be omitted.

Corollary 3. The following formula holds, for $x>0$,

$$
1-\Phi(x)=e^{-x^{2} / 2} \frac{1}{2 \pi} \int_{-\infty}^{\infty} \frac{e^{-t^{2} / 2}}{x+i t} d t
$$


For the proof it suffices to apply Lemma 3 for a standard normal random variable $X$.

Lemma 4. The following inequality holds

$$
\frac{1}{\sqrt{2 \pi}}\left(\frac{1}{x}-\frac{1}{x^{3}}\right) e^{-x^{2} / 2} \leqslant 1-\Phi(x) \leqslant \frac{1}{\sqrt{2 \pi} x} e^{-x^{2} / 2}, \quad x>0 .
$$

The proof of this well-known inequality can be found, for instance, in [7, Ch. VII, $\S 1]$.

The following result is due to Pinelis [17, p. 359].

Lemma 5. Let $X_{1}, \ldots, X_{n}$ be i.i.d. random variables such that $\mathbf{P}\left\{X_{1}= \pm 1\right\}=\frac{1}{2}$ and let $y_{1}, \ldots, y_{n}$ be real numbers such that $y_{1}^{2}+\cdots+y_{n}^{2}=1$. Then, for any $u>0$,

$$
\mathbf{P}\left\{\left|y_{1} X_{1}+\cdots+y_{n} X_{n}\right|>u\right\}<2 c(1-\Phi(u)),
$$

where $c=2 e^{3} / 9$.

Lemma 6. Let $X_{1}, \ldots, X_{n}$ denote independent random variables under assumptions of Lemma 1 . Then

$$
\sup _{x}\left|\mathbf{P}\left\{B_{n}^{-1 / 2} \sum_{j=1}^{n} X_{j}<x\right\}-\Phi(x)\right| \leqslant c L_{n} .
$$

The proof of Lemma 6 (the Berry-Esseen inequality) see in $[16, \mathrm{Ch} . \mathrm{V}$, $\S 2]$.

Our next aim is to prove the statement (1.11) of Theorem 4 for $0<x \leqslant$ $\varepsilon_{n}^{-1 / 3}$, where $\varepsilon_{n}:=L_{n}+\mathbf{P}\left\{V_{n}^{2} \leqslant B_{n}^{2} / 2\right\}$. Let us return to formula (2.4) with $D=Q$ and represent the interior integral $I(x, T), T>\frac{1}{10} L^{-1}$, in the form

$$
\begin{aligned}
I(x, T)= & \frac{1}{2 \pi}\left(\int_{-L^{-1 / 3} / 2}^{L^{-1 / 3} / 2}+\int_{L^{-1 / 3} / 2 \leqslant|t| \leqslant L^{-1} / 10}+\int_{L^{-1 / 10<|t| \leqslant T}}\right) \\
& \times \frac{e^{-i x t}}{x+i t} \prod_{j=1}^{n} \psi_{j}(t ; x) d t:=I_{1}(x)+I_{2}(x)+I_{3}(x, T) .
\end{aligned}
$$

By (2.5), we see that

$$
\frac{1}{v_{n}} \max _{j}\left|x_{j}\right| \leqslant L^{1 / 3} \leqslant \frac{1}{2(x+1)} .
$$

Estimate now the integral $I_{1}(x)$, using (2.8). Note that $|\operatorname{th} y| \leqslant y$ for all real $y$ and $|\operatorname{tg} y| \leqslant 2|y|$ for real $y$ such that $|y| \leqslant 1$, and, by (2.8), $\max \{x,|t|\} \frac{\left|x_{j}\right|}{v_{n}} \leqslant \max \left\{x, \frac{1}{2} L^{-1 / 3}\right\} L^{1 / 3} \leqslant \frac{1}{2}, \quad 2|t| x \frac{x_{j}^{2}}{v_{n}^{2}} \leqslant L^{-1 / 3} x L^{2 / 3} \leqslant \frac{1}{2}$ 
for $t \in\left[-\frac{1}{2} L^{-1 / 3}, \frac{1}{2} L^{-1 / 3}\right]$. With the help of the series expansion for the function $\ln (1+z),|z|<1$, we easily deduce the following relation for the functions $\psi_{j}(t ; x)$

$$
\begin{aligned}
\psi_{j}(t ; x) & =\cos \frac{t x_{j}}{v_{n}}\left(1+i \operatorname{th} \frac{x x_{j}}{v_{n}} \operatorname{tg} \frac{t x_{j}}{v_{n}}\right) \\
& =\cos \frac{t x_{j}}{v_{n}} \exp \left\{i \operatorname{th} \frac{x x_{j}}{v_{n}} \operatorname{tg} \frac{t x_{j}}{v_{n}}+3 \theta \frac{t^{2} x^{2} x_{j}^{4}}{v_{n}^{4}}+4 i \theta \frac{t^{3} x^{3} x_{j}^{6}}{v_{n}^{6}}\right\}
\end{aligned}
$$

for $t \in\left[-\frac{1}{2} L^{-1 / 3}, \frac{1}{2} L^{-1 / 3}\right]$. Recall that th $y=-i \operatorname{tg}(i y)$. By the inequalities $|\operatorname{th} y-y| \leqslant|y|^{3} / 2$ for all real $y$ and $|\operatorname{tg} y-y| \leqslant|y|^{3}$ for real $y$ such that $|y| \leqslant 1$, we have, for the same $t$,

$$
\operatorname{th} \frac{x x_{j}}{v_{n}} \operatorname{tg} \frac{t x_{j}}{v_{n}}=t x \frac{x_{j}^{2}}{v_{n}^{2}}+\theta t x \frac{x_{j}^{4}}{v_{n}^{4}}\left(t^{2}+\frac{1}{2} x^{2}+\frac{1}{2} t^{2} x^{2} \frac{x_{j}^{2}}{v_{n}^{2}}\right) .
$$

We obtain from (2.9) and (2.10)

$$
\prod_{j=1}^{n} \psi_{j}(t ; x)=e^{f_{1}(t ; x)+i f_{2}(t ; x)} e^{i t x} \prod_{j=1}^{n} \cos \frac{t x_{j}}{v_{n}}
$$

where

$$
\begin{aligned}
& f_{1}(t ; x)=3 \theta t^{2} x^{2} \frac{1}{v_{n}^{4}} \sum_{j=1}^{n} x_{j}^{4} \\
& f_{2}(t ; x)=\theta t x\left(t^{2}+\frac{1}{2} x^{2}\right) \frac{1}{v_{n}^{4}} \sum_{j=1}^{n} x_{j}^{4}+\frac{9}{2} \theta t^{3} x^{3} \frac{1}{v_{n}^{6}} \sum_{j=1}^{n} x_{j}^{6} .
\end{aligned}
$$

The function $\exp \left\{f_{1}(t ; x)+i f_{2}(t ; x)\right\}$ admits the following bound

$$
\begin{aligned}
\left|e^{f_{1}(t ; x)+i f_{2}(t ; x)}-1\right| \leqslant & \left|e^{f_{1}(t ; x)}-1\right|+2 e^{f_{1}(t ; x)} \sin ^{2} \frac{f_{2}(t ; x)}{2} \\
& +e^{f_{1}(t ; x)}\left|\sin f_{2}(t ; x)\right| \\
\leqslant & \left|e^{f_{1}(t ; x)}-1\right|+\frac{1}{2} e^{f_{1}(t ; x)} f_{2}^{2}(t ; x)+e^{f_{1}(t ; x)}\left|f_{2}(t ; x)\right| .
\end{aligned}
$$

By (2.8), we have

$$
\begin{array}{rlrl}
\left|f_{1}(t ; x)\right| & \leqslant \frac{3}{2} t^{2} x L \leqslant \frac{1}{4} t^{2}, & \left|e^{f_{1}(t ; x)}-1\right| \leqslant\left|f_{1}(t ; x)\right| e^{\left|f_{1}(t ; x)\right|} \leqslant c t^{2} x L e^{t^{2} / 4}, \\
\left|f_{2}(t ; x)\right| \leqslant c|t|\left(t^{2}+x^{2}\right) L, & f_{2}^{2}(t ; x) \leqslant c \frac{t^{2}\left(t^{2}+x^{2}\right)^{2}}{(1+x)^{3}} L
\end{array}
$$

for $t \in\left[-\frac{1}{2} L^{-1 / 3}, \frac{1}{2} L^{-1 / 3}\right]$. Using these bounds we arrive at the inequality

$$
\left|e^{f_{1}(t ; x)+i f_{2}(t ; x)}-1\right| \leqslant c\left(t^{2} x+|t|\left(t^{2}+x^{2}\right)+\frac{t^{2}\left(t^{2}+x^{2}\right)^{2}}{(1+x)^{3}}\right) L e^{t^{2} / 4}
$$


for the same $t$. By Lemma 2, note that

$$
\left|\prod_{j=1}^{n} \cos \frac{t x_{j}}{v_{n}}-e^{-t^{2} / 2}\right| \leqslant 16 L|t|^{3} e^{-t^{2} / 3}
$$

for $|t| \leqslant 1 /\left(4 L^{1 / 3}\right)$. Since

$$
\begin{aligned}
e^{-i t x} \prod_{j=1}^{n} \psi_{j}(t ; x)= & e^{-t^{2} / 2}+\left(\prod_{j=1}^{n} \cos \frac{t x_{j}}{v_{n}}-e^{-t^{2} / 2}\right) \\
& +\left(e^{f_{1}(t ; x)+i f_{2}(t ; x)}-1\right) \prod_{j=1}^{n} \cos \frac{t x_{j}}{v_{n}}
\end{aligned}
$$

we can write the integral $I_{1}(x)$ with the help of (2.11) and (2.12) in the form

$$
I_{1}(x)=\frac{1}{2 \pi} \int_{-\infty}^{\infty} \frac{e^{-t^{2} / 2}}{x+i t} d t+\theta\left(r_{1}+r_{2}+r_{3}\right) .
$$

Here the nonnegative quantities $r_{1}, r_{2}$, and $r_{3}$ admit the bounds

$$
\begin{aligned}
& r_{1} \leqslant c L \int_{-\infty}^{\infty} \frac{|t|^{3}}{|x+i t|} e^{-t^{2} / 3} d t \leqslant c L \\
& r_{2} \leqslant c L \int_{-\infty}^{\infty}\left(|t| x+|t|\left(t^{2}+x^{2}\right)^{1 / 2}\right. \\
& r_{3} \leqslant \frac{c}{x+1} \int_{|t|>L^{-1 / 3} / 2}^{\left.+t^{2}\left(t^{2}+x^{2}\right)^{3 / 2}(1+x)^{-3}\right) e^{-t^{2} / 12} d t \leqslant c(x+1) L},
\end{aligned}
$$

Let us estimate the integral $I_{2}(x)$. First we determine Lyapunov's ratio $L_{1}$ for the independent random variables $Y_{j}, j=1, \ldots, n$, with the characteristic functions $\psi_{j}(t ; x), j=1, \ldots, n$, respectively. Since

$$
\begin{gathered}
\mathbf{E} Y_{j}=\frac{x_{j}}{v_{n}} \operatorname{th} \frac{x x_{j}}{v_{n}}, \quad \mathbf{D} Y_{j}=\frac{x_{j}^{2}}{v_{n}^{2}}\left(1-\operatorname{th}^{2} \frac{x x_{j}}{v_{n}}\right), \\
\mathbf{E}\left|Y_{j}-\mathbf{E} Y_{j}\right|^{3}=\frac{\left|x_{j}\right|^{3}}{v_{n}^{3}}\left(1-\operatorname{th}^{4} \frac{x x_{j}}{v_{n}}\right),
\end{gathered}
$$

we have

$$
L_{1}=\left(\sum_{j=1}^{n}\left(1-\operatorname{th}^{2} \frac{x x_{j}}{v_{n}}\right) \frac{x_{j}^{2}}{v_{n}^{2}}\right)^{-3 / 2} \sum_{j=1}^{n}\left(1-\operatorname{th}^{4} \frac{x x_{j}}{v_{n}}\right) \frac{\left|x_{j}\right|^{3}}{v_{n}^{3}} .
$$

By (2.8), we see that

$$
\frac{15}{16} L \leqslant L_{1} \leqslant\left(\frac{4}{3}\right)^{3 / 2} L
$$


In addition we note that

$$
1 \geqslant \sum_{j=1}^{n} \mathbf{D} Y_{j} \geqslant 1-\frac{x^{2}}{v_{n}^{4}} \sum_{j=1}^{n} x_{j}^{4}=1-\frac{1}{2}|\theta| x L .
$$

By Lemma 2 and (2.15), (2.16), we then obtain

$$
\left|\prod_{j=1}^{n} \psi_{j}(t ; x)\right| \leqslant c\left(1+t^{2}\right) e^{-(1-x L / 2) t^{2} / 3} \leqslant c\left(1+t^{2}\right) e^{-t^{2} / 4}
$$

for $|t| \leqslant \frac{1}{10} L^{-1}$. This bound yields the inequality

$$
\left|I_{2}(x)\right| \leqslant c \int_{|t|>L^{-1 / 3} / 2} \frac{t^{2}+1}{|x+i t|} e^{-t^{2} / 4} d t \leqslant \frac{c}{x+1} L .
$$

Consider now the integral $I_{3}(x, T)$ for $T>\frac{1}{10} L^{-1}$. Represent it in the form

$$
\begin{aligned}
I_{3}(x, T)= & \frac{1}{2 \pi} \int_{L^{-1} / 10<|t| \leqslant T} \frac{e^{-i x t}}{i t} \prod_{j=1}^{n} \psi_{j}(t ; x) d t \\
& -\frac{x}{2 \pi} \int_{L^{-1} / 10<|t| \leqslant T} \frac{e^{-i x t}}{i t(x+i t)} \prod_{j=1}^{n} \psi_{j}(t ; x) d t \\
:= & I_{3,1}(x, T)+I_{3,2}(x, T) .
\end{aligned}
$$

It is easy to see that

$$
\left|I_{3,2}(x, T)\right| \leqslant \frac{x}{2 \pi} \int_{|t|>L^{-1} / 10} \frac{d t}{|t| \sqrt{x^{2}+t^{2}}} \leqslant \frac{x}{2 \pi} \int_{|t|>L^{-1} / 10} \frac{d t}{t^{2}} \leqslant \frac{5}{\pi} x L .
$$

Rewrite $I_{3,1}(x, T)$ in the form

$$
\begin{aligned}
& \frac{1}{2 \pi} \int_{-T}^{T} \frac{e^{-i x t}}{i t} \prod_{j=1}^{n}\left(\psi_{j}(t ; x)-e^{i t \mathbf{E} Y-t^{2} / 2}\right) d t \\
& \quad-\frac{1}{2 \pi} \int_{|t| \leqslant L^{-1} / 10} \frac{e^{-i x t}}{i t} \prod_{j=1}^{n}\left(\psi_{j}(t ; x)-e^{i t \mathbf{E} Y-t^{2} / 2}\right) d t \\
& \quad+\frac{1}{2 \pi} \int_{L^{-1} / 10<|t| \leqslant T} \frac{e^{-i x t}}{i t} e^{i t \mathbf{E} Y-t^{2} / 2} d t \\
& :=I_{3,1,1}(x, T)-I_{3,1,2}(x)+I_{3,1,3}(x, T),
\end{aligned}
$$

where $Y=\sum_{j=1}^{n} Y_{j}$. First we note that

$$
\lim _{T \rightarrow \infty} I_{3,1,1}(x, T)=\Phi(x-\mathbf{E} Y)-\mathbf{P}\{Y<x\} .
$$

By Lemma 6 and (2.15), (2.16),

$$
\left|\lim _{T \rightarrow \infty} I_{3,1,1}(x, T)\right| \leqslant c L_{1}+\left|\Phi\left(\frac{x-\mathbf{E} Y}{\sqrt{\mathbf{D} Y}}\right)-\Phi(x-\mathbf{E} Y)\right| \leqslant c L .
$$


By Lemma 2 and (2.15), (2.16), we get

$$
\begin{aligned}
\left|I_{3,1,2}(x)\right| \leqslant & \frac{1}{2 \pi} \int_{|t| \leqslant L^{-1} / 10}\left|\prod_{j=1}^{n} \psi_{j}(t ; x)-e^{i t \mathbf{E} Y-(\mathbf{D} Y) t^{2} / 2}\right| \frac{d t}{|t|} \\
& +\frac{1}{2 \pi} \int_{-\infty}^{\infty}\left|e^{-t^{2} / 2}-e^{-(\mathbf{D} Y) t^{2} / 2}\right| \frac{d t}{|t|} \\
\leqslant & c(x+1) L_{1} \int_{-\infty}^{\infty}\left(|t|+t^{2}\right) e^{-t^{2} / 4} d t \\
\leqslant & c(x+1) L_{1} \leqslant c(x+1) L .
\end{aligned}
$$

In addition we have $\left|I_{3,1,3}(x, T)\right| \leqslant c L$. In view of the last three bounds, we obtain from (2.19) the inequality

$$
\left|\lim _{T \rightarrow \infty} I_{3,1}(x, T)\right| \leqslant c(x+1) L .
$$

Comparing (2.18) and (2.20) we finally deduce

$$
\left|\lim _{T \rightarrow \infty} I_{3}(x, T)\right| \leqslant c(x+1) L .
$$

Now let us return to formula (2.13). By Corollary 1 and (2.13), (2.14), we have

$$
I_{1}(x)=(1-\Phi(x)) e^{x^{2} / 2}+\theta c(x+1) L .
$$

Then, in view of (2.17), (2.21), and (2.22), we obtain from (2.7)

$$
\lim _{T \rightarrow \infty} I(x, T)=(1-\Phi(x)) e^{x^{2} / 2}+\theta c(x+1) L .
$$

Using (2.8) and the series expansion for $\ln (1+z),|z|<1, \ln 1=0$, we see that, for $\left(x_{1}, \ldots, x_{n}\right) \in Q$,

$$
\begin{aligned}
\ln \operatorname{ch} \frac{x x_{j}}{v_{n}} & =\ln \left\{1+\frac{1}{2}\left(\frac{x x_{j}}{v_{n}}\right)^{2}+\frac{1}{4 !}\left(\frac{x x_{j}}{v_{n}}\right)^{4}\left(1+\frac{\theta}{20}\left(\frac{x x_{j}}{v_{n}}\right)^{2}\right)\right\} \\
& =\frac{1}{2}\left(\frac{x x_{j}}{v_{n}}\right)^{2}-\frac{1}{12}\left(\frac{x x_{j}}{v_{n}}\right)^{4}\left(1+2 \theta\left(\frac{x x_{j}}{v_{n}}\right)^{2}\right) .
\end{aligned}
$$

Using again (2.8) we obtain, for $\left(x_{1}, \ldots, x_{n}\right) \in Q$,

$$
\begin{aligned}
\prod_{j=1}^{n} \operatorname{ch} \frac{x x_{j}}{v_{n}} & =\exp \left\{\frac{x^{2}}{2}-\frac{x^{4}}{12} \sum_{j=1}^{n}\left(\frac{x_{j}}{v_{n}}\right)^{4}+\frac{\theta x^{6}}{6} \sum_{j=1}^{n}\left(\frac{x_{j}}{v_{n}}\right)^{6}\right\} \\
& =e^{x^{2} / 2}\left(1-\frac{1}{2}|\theta| x^{3} L\right) .
\end{aligned}
$$

Applying (2.23) and (2.24) to (2.4), we deduce with the help of Lemma 4 the relation

$$
\lim _{N \rightarrow \infty} I(x, N, Q)=(1-\Phi(x)) \int_{Q} d P_{1, \ldots, n}+c \theta\left(x^{2}+1\right) e^{-x^{2} / 2} \int_{Q} \theta L d P_{1, \ldots, n} .
$$


By Jensen's inequality, $\left|x_{1}\right|^{3}+\cdots+\left|x_{n}\right|^{3} \leqslant v_{n}^{3}$, therefore

$$
\begin{aligned}
\int_{Q} L d P_{1, \ldots, n} & \leqslant \mathbf{P}\left\{V_{n}^{2} \leqslant \frac{1}{2} B_{n}^{2}\right\}+2^{3 / 2} B_{n}^{-3} \int_{\mathbf{R}^{n}} \sum_{j=1}^{n}\left|x_{j}\right|^{3} d P_{1, \ldots, n} \\
& \leqslant \mathbf{P}\left\{V_{n}^{2} \leqslant \frac{1}{2} B_{n}^{2}\right\}+2^{3 / 2} L_{n} .
\end{aligned}
$$

Thus we have

$$
\lim _{N \rightarrow \infty} I(x, N, Q)=(1-\Phi(x)) \int_{Q} d P_{1, \ldots, n}+c \theta\left(x^{2}+1\right) e^{-x^{2} / 2} \varepsilon_{n} .
$$

Recall that $\varepsilon_{n}:=L_{n}+\mathbf{P}\left\{V_{n}^{2} \leqslant \frac{1}{2} B_{n}^{2}\right\}$. With the help of (2.5) we note as above that

$$
\int_{\mathbf{R}^{n} \backslash Q} d P_{1, \ldots, n} \leqslant 8(1+x)^{3} \int_{\mathbf{R}^{n} \backslash Q} L d P_{1, \ldots, n}+\mathbf{P}\left\{V_{n}=0\right\} \leqslant c\left(x^{3}+1\right) \varepsilon_{n} .
$$

Then, applying (2.26) to (2.25), we arrive, in view of Lemma 4 , at the relation

$$
\begin{aligned}
\lim _{N \rightarrow \infty} I(x, N, Q) & =1-\Phi(x)+c \theta\left(x^{2}+1\right) e^{-x^{2} / 2} \varepsilon_{n} \\
& =(1-\Phi(x))\left(1+c \theta\left(x^{3}+1\right) \varepsilon_{n}\right) .
\end{aligned}
$$

To estimate $I\left(x, N, \mathbf{R}^{n} \backslash Q\right)$ we use Lemma 5 with $y_{j}=x_{j} / v_{n}$ and $(2.1),(2.26)$. We have

$$
\begin{aligned}
\left|\lim _{N \rightarrow \infty} I\left(x, N, \mathbf{R}^{n} \backslash Q\right)\right| & \leqslant c(1-\Phi(x)) \int_{\mathbf{R}^{n} \backslash Q} d P_{1, \ldots, n} \\
& \leqslant c\left(x^{3}+1\right) \varepsilon_{n}(1-\Phi(x)) .
\end{aligned}
$$

The statement (1.11) of Theorem 4 for $0<x \leqslant \varepsilon_{n}^{-1 / 3}$ follows from (2.3) with $D=Q$ and (2.27), (2.28).

The statement (1.11) of Theorem 4 for $x>\varepsilon_{n}^{-1 / 3}$ immediately follows from Lemma 5 and formulas (2.1) and (2.2).

The statement (1.12) of Theorem 4 is an evident sequence of (1.11) and (1.10).

$\mathrm{R}$ e $\mathrm{m}$ a r k. Denote by $Q_{1}$ the set of points $\left(x_{1}, \ldots, x_{n}\right) \in \mathbf{R}^{n} \backslash\{\mathbf{0}\}$, satisfying the inequality

$$
\frac{1}{v_{n}} \max _{j}\left|x_{j}\right| \leqslant \frac{1}{2(x+1)} .
$$

Repeating the argument which we employed to deduce (2.25) and (2.28), we obtain, for $0<x \leqslant \varepsilon_{n}^{-1 / 3}$, the following formula

$$
\begin{aligned}
1-\mathbf{P} & \left\{\frac{S_{n}}{V_{n}}<x\right\}=(1-\Phi(x)) \int_{Q_{1}} d P_{1, \ldots, n} \\
& -(1-\Phi(x)) \int_{Q_{1}}\left(1-\exp \left\{-\frac{x^{4}}{12}\left(1+\frac{\theta}{2}\right) \sum_{j=1}^{n}\left(\frac{x_{j}}{v_{n}}\right)^{4}\right\}\right) d P_{1, \ldots, n} \\
& +c \theta(1-\Phi(x)) \int_{\mathbf{R}^{n} \backslash Q_{1}} d P_{1, \ldots, n}+c\left(x^{2}+1\right) \varepsilon_{n}(1-\Phi(x)) .
\end{aligned}
$$


$\mathrm{Pr}$ o of of $\mathrm{T}$ h e or e $\mathrm{m} 2$. Note that the random variables under assumptions of Theorem 2 satisfy the assumptions of Theorem 4 . Therefore (1.11) holds. In view of Lemma $1, \mathbf{P}\left\{V_{n}^{2} \leqslant \frac{1}{2} B_{n}^{2}\right\} \leqslant c L_{n}$ and we obtain the statement of the theorem from (1.11).

$\mathrm{Pr}$ o of of Proposition. In the proof of Proposition we continue to use the notation of Section 2. Let $x \in\left(0, n^{1 / 6} / b_{n}^{1 / 3}\right]$ and $\sqrt{n} / b_{n} \geqslant c$, where $c$ is sufficiently large. Consider symmetric i.i.d. random variables $X_{1}, \ldots, X_{n}$ such that

$$
\mathbf{P}\left\{X_{1}=a\right\}=\frac{1}{2}-\frac{b_{n}(x+1)^{3}}{4 n^{3 / 2}}, \quad \mathbf{P}\left\{X_{1}=\frac{a \sqrt{n}}{2(x+1)}\right\}=\frac{b_{n}(x+1)^{3}}{4 n^{3 / 2}}
$$

where

$$
a=a(n, x):=\left(1-\frac{b_{n}}{2 \sqrt{n}}\left(\frac{(x+1)^{3}}{n}-\frac{(x+1)}{4}\right)\right)^{-1 / 2} .
$$

Denote the distribution of these random variables by $P$. It is easy to see that $\mathbf{E} X_{1}^{2}=1$ and $c_{1} b_{n} \leqslant \mathbf{E}\left|X_{1}\right|^{3} \leqslant c_{2} b_{n}$.

Consider vectors $\left(x_{1}, \ldots, x_{n}\right) \in \mathbf{R}^{n}$ such that $x_{j}= \pm a, \pm a \sqrt{n} /(2(x+1))$ for $j=1, \ldots, n$. In the proof of proposition we will consider such vectors only. By the simple estimate $v_{n}^{2} \geqslant n a^{2}$, we see that $v_{n}^{-1} \max _{j}\left|x_{j}\right| \leqslant$ $(2(x+1))^{-1}$ for all considered $\left(x_{1}, \ldots, x_{n}\right)$. Thus in our case $Q_{1}=\mathbf{R}^{n}$ and we obtain from (2.29), taking into account Lemma 1 ,

$$
\begin{aligned}
& \frac{1-\mathbf{P}\left\{S_{n} / V_{n}<x\right\}}{1-\Phi(x)}=1+c \theta\left(x^{2}+1\right) \frac{b_{n}}{\sqrt{n}} \\
& -\int_{\mathbf{R}^{n}}\left(1-\exp \left\{-\frac{x^{4}}{12}\left(1+\frac{\theta}{2}\right) \sum_{j=1}^{n}\left(\frac{x_{j}}{v_{n}}\right)^{4}\right\}\right) d P \times \cdots \times P .
\end{aligned}
$$

Denote by $Q_{2}$ the set of vectors $\left(x_{1}, \ldots, x_{n}\right)$ such that only one of $x_{j}$, $j=1, \ldots, n$, takes the values $\pm a \sqrt{n} /(2(x+1))$. It is easy to see that

$$
\int_{Q_{2}} d P \times \cdots \times P=\left(1-\frac{b_{n}(x+1)^{3}}{2 n^{3 / 2}}\right)^{n-1} \frac{b_{n}(x+1)^{3}}{2 n^{1 / 2}} \geqslant \frac{b_{n}(x+1)^{3}}{8 n^{1 / 2}} .
$$

Since $v_{n}^{2} \leqslant 5 n a^{2} / 4$, for $\left(x_{1}, \ldots, x_{n}\right) \in Q_{2}$, we have

$$
x^{4} \sum_{j=1}^{n}\left(\frac{x_{j}}{v_{n}}\right)^{4} \geqslant \frac{1}{32}, \quad\left(x_{1}, \ldots, x_{n}\right) \in Q_{2} .
$$

Using (2.31) and (2.32), we obtain from (2.30)

$$
\frac{1-\mathbf{P}\left\{S_{n} / V_{n}<x\right\}}{1-\Phi(x)} \leqslant 1-c_{3}\left((x+1)^{3}-c\left(x^{2}+1\right)\right) \frac{b_{n}}{\sqrt{n}},
$$

as was to be proved. 


\section{REFERENCES}

1. Bentkus V., Götze F. The Berry-Esseen bound for Student's statistic. - Ann. Probab., 1996, v. 24, № 1, p. 491-503.

2. Bentkus V., Bloznelis M., Götze F. A Berry-Esseen bound for Student's statistic in the non-i.i.d. case. - J. Theoret. Probab., 1996, v. 9, № 3, p. 765-796.

3. Chistyakov G. P., Götze F. Moderate deviations for self-normalized sums. Preprint SFB 343. 99-048. Bielefeld: Universität Bielefeld, 1999, p. 1-36.

4. Csörgö S., Mason D. M. Approximations of weighted empirical processes with applications to extreme, trimmed and self-normalized sums. - Proceedings of the First World Congress of the Bernoulli Society (Tashkent, 1986). V. 2. Utrecht: VNU Sci. Press, 1987 , p. $811-819$.

5. Efron B. Student's $t$-test under symmetry conditions. - J. Amer. Statist. Assoc., 1969 , v. 64 , p. $1278-1302$.

6. Егоров $B$. А. Об асимптотическом поведении самонормированных сумм случайных величин. - Теория вероятн. и ее примен., 1996, т. 41, с. 643-650.

7. Феллер В. Введение в теорию вероятностей и ее приложения. Т. 1. М.: Мир, 1984, $528 \mathrm{c}$.

8. Giné E., Götze F., Mason D. M. When is the Student $t$-statistic asymptotically standard normal. - Ann. Probab., 1997, v. 25, № 3, p. 1514-1531.

9. Griffin P. S., Mason D. M. On the asymptotic normality of self-normalized sums. Math. Proc. Cambridge Philos. Soc., 1991, v. 109, № 3, p. 597-610.

10. Hall $P$. On the effect of random norming on the rate of convergence in the central limit theorem. - Ann. Probab., 1987, v. 16, № 3, p. 1265-1280.

11. Horváth L., Shao Q. M. Large deviations and law of the iterated logarithm for partials sums normalized by the largest absolute observation. - Ann. Probab., 1996, v. 24, № 3, p.1368-1387

12. Ибрагимов И.А., Линник Ю.В. Независимые и стационарно-связанные величины. М.: Наука, 1965, 524 с.

13. Линник Ю.В. Предельные теоремы для сумм независимых величин при учете больших уклонений. - Теория вероятн. и ее примен., 1961, т. 6, в. 2, с. 145-163.

14. Logan B., Mallows C., Rice S., Shepp L. Limit distributions of self-normalized sums. - Ann. Probab., 1973, v. 1, p. 788-809.

15. Maller R. A. A theorem on products of random variables, with application to regression. - Austral. J. Statist., 1981, v. 23, p. 177-185.

16. Петров В. В. Суммы независимых случайных величин. М.: Наука, 1972, 414 с.

17. Pinelis $I$. Extremal probabilistic problems and Hotelling's $T^{2}$ test under a symmetry condition. - Ann. Statist., 1994, v. 22, p. 357-368.

18. Shao Q.-M. Self-normalized large deviations. - Ann. Probab., 1997, v. 25, № 1, p. $285-328$.

19. Shao Q.-M. Recent development in self-normalized limit theorems. - Asymptotic Methods in Probability and Statistics. Ed. by B. Szyszkowicz. Amsterdam: NorthHolland/Elsevier, 1998, p. 467-480.

20. Shao Q.-M. A Cramér type large deviation result for Student's $t$-statistic. - J. Theoret. Probab., 1999 , v. 12, № 2, p. 385-398.

21. Шарахметов ШІ. Неравенство Берри-Эссеена для статистики Стьюдента. - Узб. матем. журн., 1995, № 2, с. 101-112.

22. Slavova V. V. On the Berry-Esseen bound for Student's statistic. - Lecture Notes in Math., 1985, v. 1155, p. 335-390.

23. Wang Q., Jing B.-Y. An exponential nonuniform Berry-Essen bound for selfnormalized sums. - Ann. Probab., 1999, v. 27, № 4, p. 2068-2088.

Поступила в редакцию 24.IX.2001 